\title{
Application of contrast-enhanced ultrasonography in the diagnosis of post- kidney transplant lymphoproliferative disorder in native kidney- a case report
}

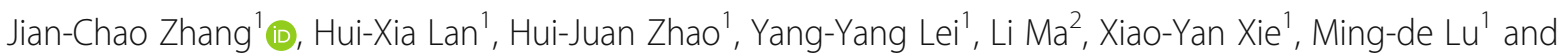
Wei Wang ${ }^{1 *}$ (D)

\begin{abstract}
Background: Post-transplant lymphoproliferative disorders (PTLDs) represent a spectrum of heterogenetic lymphoid proliferations. PTLD is a serious complication that affects the long-term survival of kidney transplant patients. Imaging examination is an important method for detecting and diagnosing PTLD. Contrast-enhanced ultrasonography (CEUS) and CEUS-guided biopsy are important modalities for tumor detection and diagnosis. In this case, we describe a 69 years old man in whom a native kidney PTLD was confirmed by CEUS.

Case presentation: A 69-year-old male patient who had a kidney transplant 1 year earlier presented with 3 months of progressive myasthenia of both lower limbs associated with amyotrophy and weight loss. Although positron emission tomography/computed tomography (PET-CT) showed a high metabolic lesion in the untransplanted kidney, abdominal contrast enhanced computed tomography cannot detect the lesion in the atrophic left kidney. The above examinations showed that the transplanted kidney was normal. CEUS can detect a homogeneously enhanced lesion in the same location as PET-CT. Subsequently, a biopsy was performed under CEUS guidance, and the final pathological diagnosis was diffuse large B-cell lymphoma. The patient then received the R-CHOP treatment. Unfortunately, pulmonary thromboembolism occurred 2 weeks later, and the patient's condition was not alleviated through active treatment. Finally, the patient's family gave up treatment, and the patient was discharged.
\end{abstract}

Conclusion: The case suggested that CEUS was a valuable imaging method for patient with renal transplantation to detect and diagnose of PTLD.

Keywords: Contrast-enhanced ultrasonography, Biopsy, Post transplantation lymphoproliferative, Kidney transplantation

\section{Background}

Post-transplant lymphoproliferative disorders (PTLDs) represent a group of heterogenetic lymphoid proliferations ranging from polyclonal lymphoid proliferation to lymphomas. PTLD occurs after solid organ transplantation and is associated with administration of

\footnotetext{
*Correspondence: wangw73@mail.sysu.edu.cn

${ }^{1}$ Department of Medical Ultrasonics, Institute of Diagnostic and

Interventional Ultrasound, The First Affiliated Hospital of Sun Yat-Sen

University, No.58 Zhongshan Road 2, Guangzhou 510080, People's Republic of China

Full list of author information is available at the end of the article
}

immunosuppressive agents [1]. Prompt diagnosis of PTLD is critical to prognosis, to prevent the further development of malignant lymphoma [2]. Imaging examination is an important method to detect and diagnose PTLD, including conventional ultrasonography (US), Doppler ultrasound, computed tomography (CT) and magnetic resonance imaging (MRI). Contrast-enhanced computed tomography (CE-CT) and contrast-enhanced magnetic resonance imaging (CE-MRI) are important image modalities for characterizing PTLD [3, 4]. However both the iodinated contrast agent for CE-CT and

(c) The Author(s). 2019 Open Access This article is distributed under the terms of the Creative Commons Attribution 4.0 International License (http://creativecommons.org/licenses/by/4.0/), which permits unrestricted use, distribution, and reproduction in any medium, provided you give appropriate credit to the original author(s) and the source, provide a link to the Creative Commons license, and indicate if changes were made. The Creative Commons Public Domain Dedication waiver (http://creativecommons.org/publicdomain/zero/1.0/) applies to the data made available in this article, unless otherwise stated. 
the gadolinium for MRI have potentially nephrotoxic, restricting their use in patients with impaired renal function [5]. Compared with CE-MRI and CE-CT, the contrast agents used for contrast-enhanced ultrasonography (CEUS) are no nephrotoxic and can be safety applied to patients with renal dysfunction. CEUS can also provide real-time visualization of contrast-enhanced patterns, which can be used for differential diagnosis of renal lesions [6]. In addition, the exact real-time aspect of CEUS makes it uniquely suited for interventions [7]. CEUS therefore has gradually become the preferred method to detect and diagnose renal tumors in chronic kidney disease and post-transplant patients in recent years $[8,9]$. We report a case of a 69 year old man with a native renal lymphoma associated with PTLD. We describe the contrast-enhanced features of the tumor and the application of a CEUS guided biopsy on the tumor, which is not well visualized using US. To the best of our knowledge this is the first report on CEUS manifestations of native renal lymphoma following renal transplantation.

\section{Case presentation}

A 69-year-old male patient with kidney transplantation was submitted to our hospital for further evaluation and treatment of a left native kidney mass. The patient had chronic kidney disease for 2 years and had undergone dialysis for 10 months before kidney transplantation. The patient had a 20 years history of hypertension (the highest blood pressure: 170/106 $\mathrm{mmHg}$ ) without diabetes. The patient underwent kidney transplantation 11 months prior to our study, and maintained a triple immunosuppressive regimen that consisted of tacrolimus, mycophenolatemofetil and prednisolone after transplantation. Two months prior, the patient was submitted to the local hospital for progressive myasthenia both lower limbs and weight loss. Routine laboratory tests showed the following: creatinine and urea were normal, but
Epstein-Barr virus (EBV) and cytomegalovirus (CMV) IgG antibodies were positive. An MRI of the lumbar showed multiple vertebral bone destruction. A positron emission tomography/computed tomography (PET-CT) scan was then performed for further information, showing an intense FDG accumulation lesion (approximately $1.4 \mathrm{~cm}$, standardized uptake values max: 4.7 ) in the medial portion of the left untransplanted kidney (Fig. 1a). PET-CT then proposed possible diagnosis of a renal malignant tumor and bone metastasis, but it could not confirm diagnosis. CE-CT was performed with following scanning parameters: tube voltage, $120 \mathrm{kV}$; tube current, $250 \mathrm{~mA}$; and slice thicknesses, $1 \mathrm{~mm}$ and $10 \mathrm{~mm}$. CE-CT (Fig. 1b) and US detect multiple cysts only and cannot find a solid lesion proposed by PET-CT. PET-CT and CE-CT did not reveal any abnormalities in transplanted kidney. To further evaluate the tumor and definite diagnosis, the patient was sent to our department.

Ultrasonography was performed using the Aplio500 (Toshiba Medical Systems, Tokyo, Japan) equipped with a 375BT convex transducer (frequency range 3.0-6.0 $\mathrm{MHz})$. The transplanted kidney was located in the right iliac fossa, and showed no abnormalities in conventional B-mode and Doppler ultrasonography. Only atrophic untransplanted kidneys with multiple cystic lesions were observed in US (Fig. 2a). Then a bolus of $2.4 \mathrm{ml}$ of SonoVue (Bracco, Milan, Italy) was administered intravenously and flushed by $5.0 \mathrm{ml}$ of $0.9 \%$ saline was performed. The examination was performed at a low mechanical index of 0.09 . CEUS revealed a completely homogeneously enhancement lesion (approximately $1.3 \times 1.1 \mathrm{~cm})$ in the medial portion of the left untransplanted kidney in $33 \mathrm{~s}$ post-injected of contrast agent. Enhancement had progressed from the periphery towards the center of the lesion at $20 \mathrm{~s}$ post-injection (Fig. 2b). The lesion exhibited completely enhancement at $33 \mathrm{~s}$ (Fig. 2c) and was slightly higher enhanced than

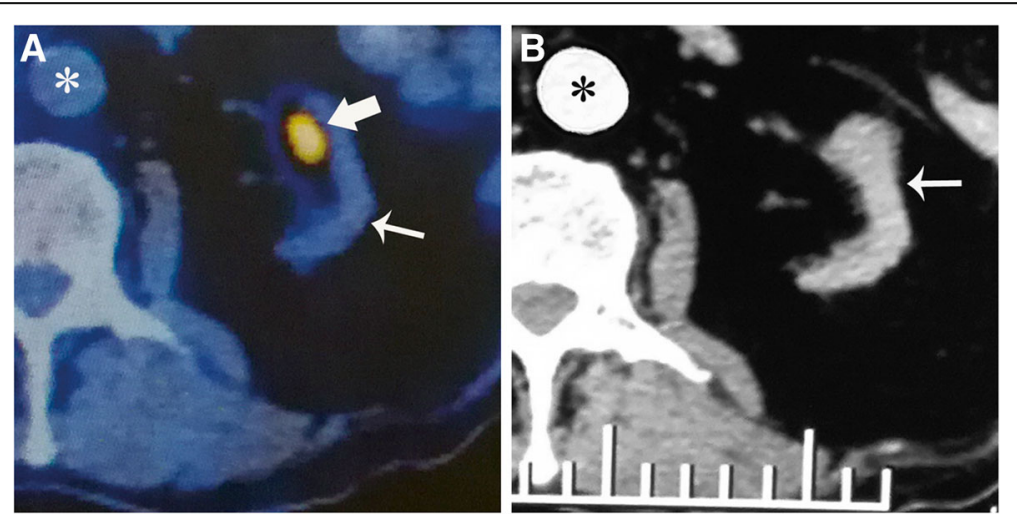

Fig. 1 Positron emission tomography/computed tomography (PET-CT) and computed tomography (CT) images of the outer hospital. a PET-CT revealed a $1.4 \mathrm{~cm}$ intense FDG uptake (Standardize Uptake Values max: 4.7) lesion (wide arrow) at the medial portion of the left native kidney; $\mathbf{b}$ AS the same level of PET-CT, contrast-enhanced CT did not detect any solid lesion in the left native kidney (narrow arrow) 
the surrounding parenchymal. The lesion gradually turned to hypo-enhancement at $90 \mathrm{~s}$ (Fig. 2d). These features of CEUS suggested a diagnosis of renal malignancy. Subsequently a biopsy was performed under CEUS guidance because of poor differentiation between the target and adjacent cysts in US. Then a further bolus of agent was injected, and a BARD automatic biopsy gun with an 18G percutaneous core needle biopsy was repeated under CEUS guidance to enhanced lesion (Fig. 3). Two tissue core samples with $2 \mathrm{~cm}$ in length were obtained from the targeted area (Fig. 4a). Histological examination of the specimen provided a diagnosis of diffuse large B-cell lymphoma (DLBCL) (Fig. 4b, c) with immunohistochemistry staining showing positive results for CD20, CD79, Bcl-2 and Bcl-6. Finally, clinical diagnosis was non-Hodgkin's lymphoma (diffuse large cell, stage III B). Clinicians consider the prognosis very poor and communicate effectively with patients and their families. The patient consented to accepted chemotherapy consisting of rituximab and cyclophosphamide, hydroxydaunomycin, oncovin and prednisolone at the standard dose. Unfortunately, the patient developed deep vein thrombosis in the right side of his lower limb and pulmonary thromboembolism 2 weeks after one cycle chemotherapy. Despite active treatment, the patient's condition has not been alleviated. Finally, the patient's family gave up treatment and the patient was discharged.

\section{Discussion and conclusions}

The incidence of PTLD in kidney transplant patients is approximately $1-10 \%$, and it occurs most frequently during the first year after transplantation. Immunosuppression and EBV infection, are two major factors associated with the progression of PTLD [2] .The Gastrointestinal tract, allograft kidney and abdominal cavity are common sites of PTLD, but it is rare in the native kidney, which has been described in only three studies [10-12]. In previous reports, both US and CECT can clearly show the tumor and be used to characterize the tumor. The tumors showed persistent hypo-enhancement throughout the examination with CE-CT. The enhancement pattern of the tumor is the main difference from clear cell renal cell carcinoma, which present avid early hyper-enhancement and early wash-out throughout the examination $[13,14]$.
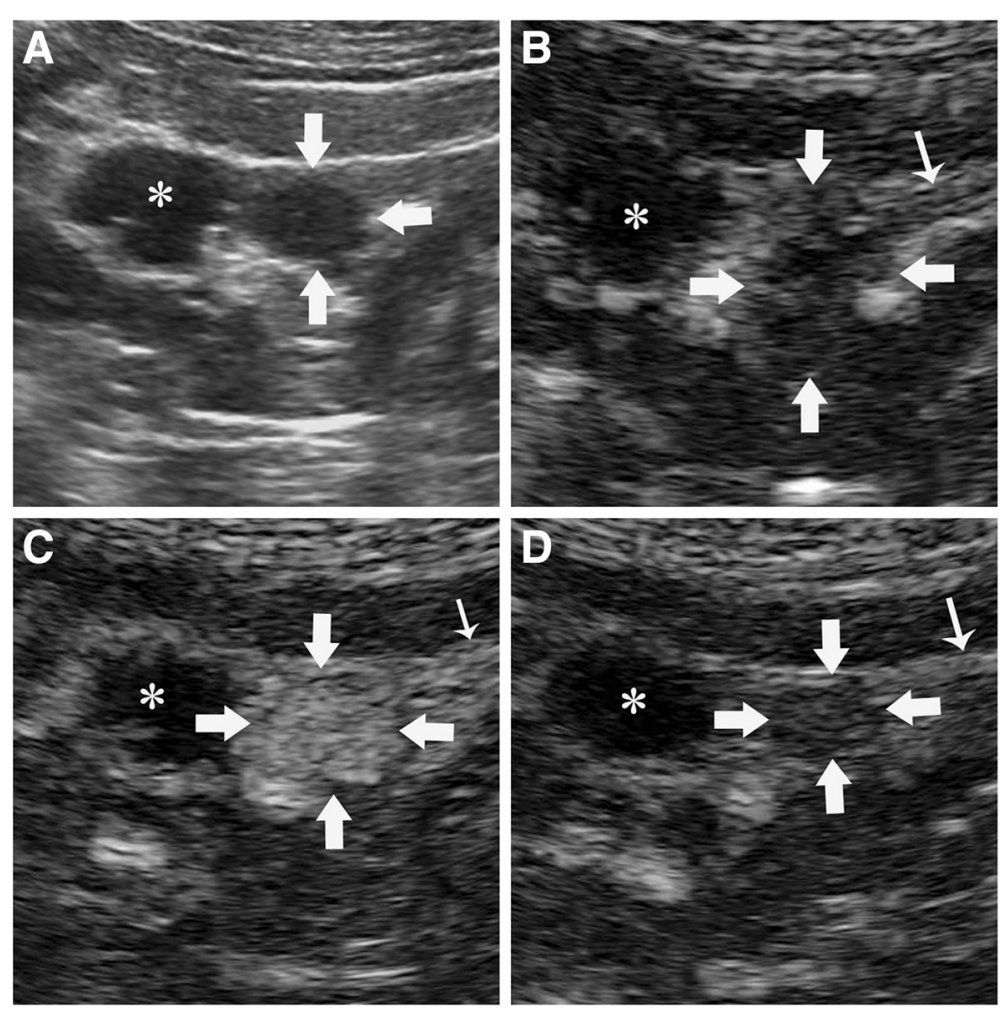

Fig. 2 The contrast-enhanced ultrasonography features of the tumor in native left kidney a the tumor (arrow) and cysts (asterisk) are presenting hypoechoic lesions in conventional ultrasonography which is difficult to differentiate from surrounding cysts. $\mathbf{b}$. After injection of contrast agent, the edge of tumor (wide arrow) start enhancing at 20 s, showing hypo-enhancement as surrounding parenchyma. c The lesion achieved peakenhancement at $33 \mathrm{~s}$, more intense than surrounding kidney parenchyma (narrow arrow). $\mathbf{d}$ The lesion gradually turned to hypo-enhancement at 90 s, showing hypo-enhancement in comparison to surrounding parenchyma. The cyst (asterisk) around the lesion still non-enhancing during whole examination 


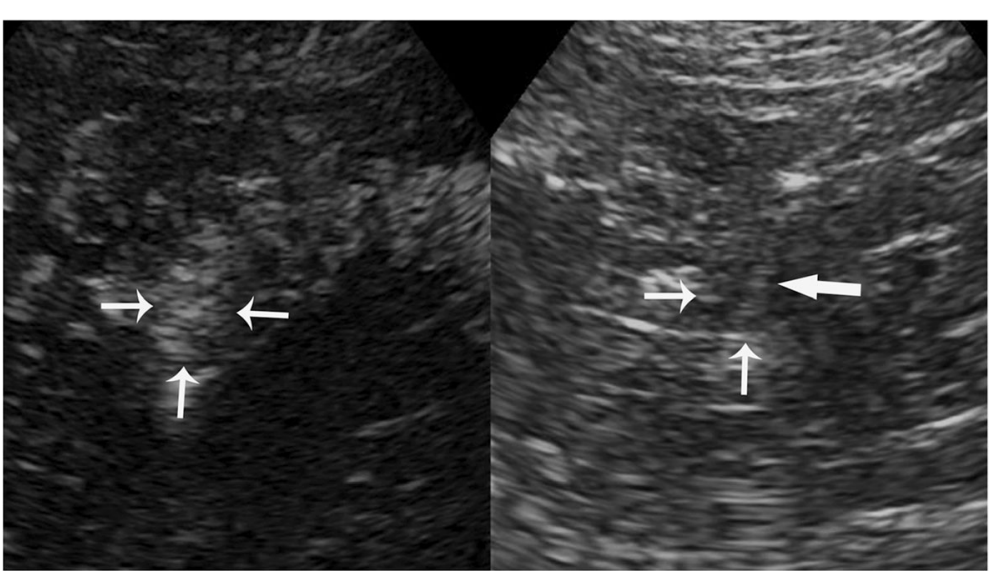

Fig. 3 Contrast-enhanced ultrasonography guided biopsy of the mass. The needle tip (wide arrow) has correctly been inserted into the completely enhanced mass (narrow arrow)

In our case, the tumor showed avid early hyper enhancement on CEUS; however, this was not visible on US and CE-CT. There are three possible explanations for this difference in our case: First, since the microvasculature of the atrophic renal parenchyma was reduced compared to a normal kidney, less iodine contrast agent entered the renal parenchyma [13]. In addition, the tumor was small in size, and there was much less iodine contrast agent entering the tumor. As a result, the tumor presented no enhancement on CE-CT, which caused a failure to distinguish tumors from surrounding cysts. Second, because CEUS contrast material is purely intravascular, it better correlates with the microvessel density of a tumor. Therefore, CEUS is even more sensitive for detection of hypovascular lesions than contrastenhanced CT [15]. Although the native kidney perfusion reduced in our case, the microvascular density of the tumor is relatively more abundant than the peripheral renal parenchyma. As a result, the tumor appeared hyper enhanced compare to the surrounding renal parenchyma on CEUS. Third, the CE-CT scan often started at $35 \mathrm{~s}$ after the beginning of the injection, but the lesion showed peak enhancement at $33 \mathrm{~s}$ during CEUS examination. This may result in missing the peak enhancement phase of the tumor on CE-CT. In contrast, real-time CEUS can continually provide information about blood
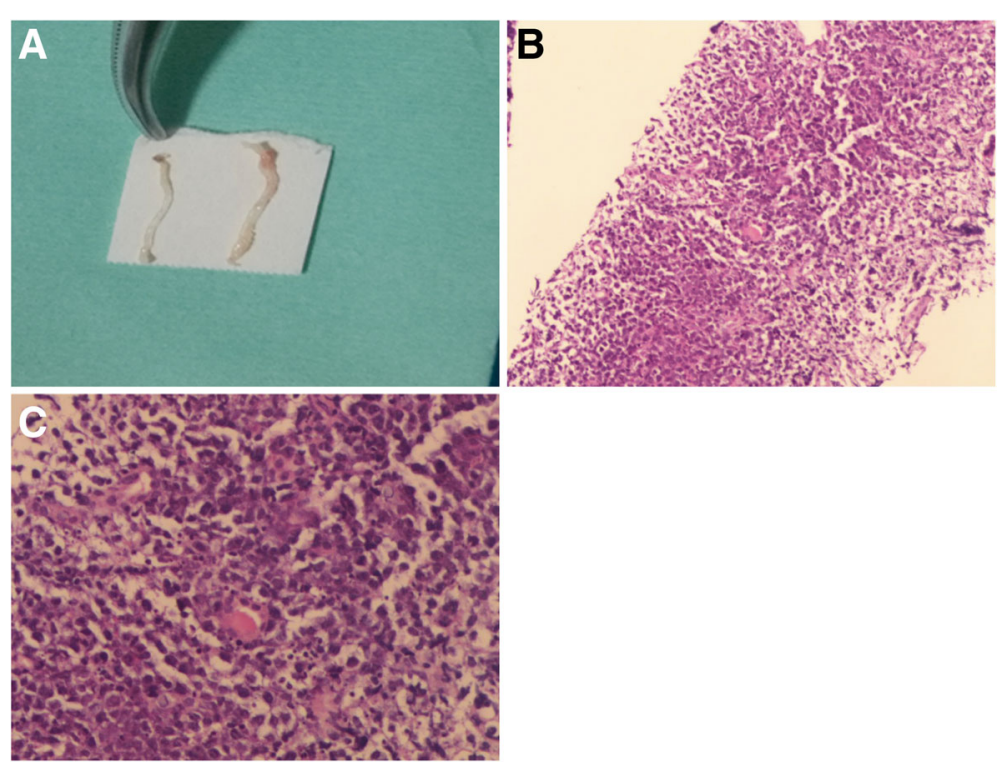

Fig. 4 Histological findings of the core needle biopsy specimen from the mass. a The core needle biopsy specimen from the mass; $\mathbf{b}$, $\mathbf{c}$ Hematoxylin and eosin staining of specimen from the mass revealed diffuse infiltration of atypical lymphoid cells. (B 10x,C 40x) 
perfusion for the renal lesion after injection, which can offer more diagnostic clues to differential the tumor from adjacent cysts.

US-guided biopsy is a common clinical method to obtain tissue specimens for histopathological analysis [16]. However, this technique may be unsuccessful when the tumor is poorly differentiated from adjacent structures. CEUS is well placed to address this problem because of its capacity to differentiate between the altered vascularization of a tumor and surrounding structures. CEUS could help further confirm the tumor border and guide the needle to the target area. CEUS also can be used to differentiate enhanced active area from nonenhanced necrotic area. By directing the biopsy needle toward enhanced areas of the lesion, the sample from necrotic parts of the lesion can be reduced [17]. In a previous report, using the contrast agent, the lesion detection rate was increased from $77.3 \%$ with US to $92.0 \%$ with CEUS during the biopsy, with a 95.2\% success rate for CEUS-guided biopsies of these lesions $[18,19]$. According to these finding, we performed the biopsy in our patient under the guidance of CEUS. Finally, the pathology of the biopsy specimen confirmed the diagnosis of diffuse large B cell lymphoma.

In summary, CEUS can provide more useful information than CE-CT to detect and diagnose PTLDs derived from atrophic native kidneys; CEUS-guided biopsy can improve the diagnostic accuracy and success rate of percutaneous biopsy. We believe that CEUS and CEUSguided biopsy may be an effective method for early screening and diagnosis of native kidney PTLD in kidney transplant patients.

\section{Abbreviations}

CE-CT: Contrast-enhanced computed tomography; CE-MRI: Magnetic resonance imaging; CEUS: Contrast-enhanced ultrasonography; CMV: Cytomegalovirus; DLBCL: Diffuse large B-cell lymphoma; EBV: EpsteinBarr virus; FDG: Fluorodeoxyglucose F18; PET-CT: Positron emission tomography/computed tomography; PTLD: Post-transplant lymphoproliferative disorders

\section{Acknowledgements}

Not applicable.

\section{Description}

Our report described the imaging feature of native kidney lymphoma on contrast-enhanced ultrasonography (CEUS) in a post-kidney transplant patient. We found that the lymphoma showed different enhanced pattern in atrophic kidney from the normal kidney. Then, we performed the tumor biopsy under the guidance of CEUS

\section{Publication of clinical datasets}

Not applicable.

\section{Authors' contributions}

$J C Z$ design the study and drafting of the manuscript; HXL, HJZ, YYL acquisition the data and images; LM designed and generated all of the submitted Figs; XYX and MDL analysis the data and images; WW interacted with the patient; made substantial contributions to the content and design of the study; critical revision of the manuscript and take accountable for all aspects of the work to ensure the reliability and accuracy of the data. All authors and all authors have made significant contribution to the content of this paper. All authors read and approved the final manuscript.

\section{Funding}

Our work is supported by National Natural Science Foundation of China (No.81701701) (to W.W), Natural Science Foundation of Guangdong Province (No.2016A030310143) (to W.W) and Department of Finance of Guangdong Province (No.20160904) (to W.W). All of the above funding was used to conduct laboratory analyses and cover publication fees.

\section{Availability of data and materials}

All data generated or analysed during this study are included in this published article.

\section{Ethics approval and consent to participate}

Not applicable.

\section{Consent for publication}

The patient provided written informed consent to publish this report and associated images. We have de-identified demographic information and other specific information of the patient.

\section{Competing interests}

Our manuscript has been read and approved by all authors and there are no any financial or other interests in the subject matter of the manuscript.

\section{Author details}

${ }^{1}$ Department of Medical Ultrasonics, Institute of Diagnostic and Interventional Ultrasound, The First Affiliated Hospital of Sun Yat-Sen University, No.58 Zhongshan Road 2, Guangzhou 510080, People's Republic of China. ' ${ }^{2}$ Department of Medical Ultrasonics, Guangdong Second People's Hospital, Haizhu District, Guangzhou 510317, People's Republic of China.

Received: 2 May 2019 Accepted: 11 November 2019

Published online: 21 November 2019

\section{References}

1. Caillard S, Dharnidharka V, Agodoa L, Bohen E, Abbott K. Posttransplant lymphoproliferative disorders after renal transplantation in the United States in era of modern immunosuppression. Transplantation. 2005;80(9):1233-43.

2. Opelz G, Döhler B. Lymphomas after solid organ transplantation: a collaborative transplant study report. Am J Transplant. 2004;4(2):222-30.

3. Ginat DT, Purakal A, Pytel P. Susceptibility-weighted imaging and diffusionweighted imaging findings in central nervous system monomorphic B cell post-transplant lymphoproliferative disorder before and after treatment and comparison with primary B cell central nervous system lymphoma. J NeuroOncol. 2015;125(2):297-305.

4. Pickhardt PJ, Siegel MJ. Posttransplantation lymphoproliferative disorder of the abdomen: CT evaluation in 51 patients. Radiology. 1999;213(1):73-8.

5. Abu JB, Leonard AC, Sharma Y, Katipally S, Shields AR, Alloway RR, Woodle ES, Thakar CV. Contrast-induced nephropathy in renal transplant recipients: a single center experience. Front Med (Lausanne). 2017;4:64.

6. Sparchez Z, Radu P, Sparchez M, Crisan N, Kacso G, Petrut B. Contrast enhanced ultrasound of renal masses. A reappraisal of EFSUMB recommendations and possible emerging applications. Med Ultrason. 2015; 17(2):219-26.

7. Liu BX, Huang GL, Xie XH, Zhuang BW, Xie XY, Lu MD. Contrast-enhanced US-assisted percutaneous nephrostomy: a technique to increase success rate for patients with nondilated renal collecting system. Radiology. 2017; 285(1):293-301.

8. Chang EH, Chong WK, Kasoji SK, Fielding JR, Altun E, Mullin LB, Kim Jl, Fine JP, Dayton PA, Rathmell WK. Diagnostic accuracy of contrast-enhanced ultrasound for characterization of kidney lesions in patients with and without chronic kidney disease. BMC Nephrol. 2017;18(1):266

9. Stock K, Kubler H, Maurer T, Slotta-Huspenina J, Holzapfel K. CEUS-diagnosis of solid renal tumors. Der Radiologe. 2018;58(6):553-62.

10. Schultz TD, Zepeda N, Moore RB. Post-transplant lymphoproliferative disorder and management of residual mass post chemotherapy: case report. Int J Surg Case Rep. 2017;38:115-8.

11. Lee M, Kim SK, Chung YE, Choi JY, Park MS, Lim JS, Kim MJ, Kim H. Necrotic lymphoma in a patient with post-transplantation lymphoproliferative 
disorder: ultrasonography and $C T$ findings with pathologic correlation Ultrasonography. 2015;34(2):148-52

12. Chandra A, Kaul A, Aggarwal V, Srivastava D. Native kidney posttransplant lymphoproliferative disorder in a renal transplant recipient. Saudi J Kidney Dis Transpl. 2017;28(4):942-4.

13. Ferda J, Hora M, Hes O, Reischig T, Kreuzberg B, Mirka H, Ferdova E, Ohlidalova K, Baxa J, Urge T. Computed tomography of renal cell carcinoma in patients with terminal renal impairment. Eur J Radiol. 2007;63(2):295-301.

14. Trenker C, Neesse A, Gorg C. Sonographic patterns of renal lymphoma in Bmode imaging and in contrast-enhanced ultrasound (CEUS)--a retrospective evaluation. Eur J Radiol. 2015;84(5):807-10.

15. Quaia E, Bertolotto M, Cioffi V, Rossi A, Baratella E, Pizzolato R, Cov MA. Comparison of contrast-enhanced sonography with unenhanced sonography and contrast-enhanced $\mathrm{CT}$ in the diagnosis of malignancy in complex cystic renal masses. AJR Am J Roentgenol. 2008;191(4):1239-49.

16. Sheafor DH, Paulson EK, Simmons CM, DeLong DM, Nelson RC. Abdominal percutaneous interventional procedures: comparison of $\mathrm{CT}$ and US guidance. Radiology. 1998;207(3):705-10.

17. Partovi S, Lu Z, Kessner R, Yu A, Ahmed Y, Patel IJ, Nakamoto DA, Azar N Contrast enhanced ultrasound guided biopsies of liver lesions not visualized on standard B-mode ultrasound-preliminary experience. J Gastrointest Oncol. 2017:8(6):1056-64.

18. Nolsoe CP, Nolsoe AB, Klubien J, Pommergaard HC, Rosenberg J, Meloni MF, Lorentzen $\mathrm{T}$. Use of ultrasound contrast agents in relation to percutaneous interventional procedures: a systematic review and pictorial essay. J Ultrasound Med. 2018;37(6):1305-24.

19. Sparchez Z, Radu P, Kacso G, Sparchez M, Zaharia T, Al HN. Prospective comparison between real time contrast enhanced and conventional ultrasound guidance in percutaneous biopsies of liver tumors. Med Ultrason. 2015;17(4):456-63.

\section{Publisher's Note}

Springer Nature remains neutral with regard to jurisdictional claims in published maps and institutional affiliations.

Ready to submit your research? Choose BMC and benefit from:

- fast, convenient online submission

- thorough peer review by experienced researchers in your field

- rapid publication on acceptance

- support for research data, including large and complex data types

- gold Open Access which fosters wider collaboration and increased citations

- maximum visibility for your research: over $100 \mathrm{M}$ website views per year

At $\mathrm{BMC}$, research is always in progress.

Learn more biomedcentral.com/submissions 